\title{
Constraining the physical properties of Titan's empty lake basins using nadir and off-nadir Cassini RADAR backscatter
}

\author{
R.J. Michaelides ${ }^{\mathrm{a}, *}$, A.G. Hayes ${ }^{\mathrm{a}}$, M. Mastrogiuseppe ${ }^{\mathrm{a}}$, H.A. Zebker ${ }^{\mathrm{b}}$, T.G. Farr ${ }^{\mathrm{c}}$, M.J. Malaska ${ }^{\mathrm{c}}$, V. Poggiali ${ }^{\mathrm{d}}$, J.P. \\ Mullen ${ }^{\mathrm{a}}$ \\ ${ }^{a}$ Department of Astronomy, Cornell University, Ithaca NY 14850 \\ ${ }^{b}$ Department of Geophysics and Electrical Engineering, Stanford University, Stanford, CA 94305 \\ ${ }^{c}$ Jet Propulsion Laboratory, Pasadena, Ca 91109 \\ ${ }^{d}$ Dipartimento di Ingegneria dell 'Informazione, Elettronica e Telecomunicazioni, Università La Sapienza, Rome, Italy
}

\begin{abstract}
We use repeat synthetic aperture radar (SAR) observations and complementary altimetry passes acquired by the Cassini spacecraft to study the scattering properties of Titan's empty lake basins. The best-fit coefficients from fitting SAR data to a quasi-specular plus diffuse backscatter model suggest that the bright basin floors have a higher dielectric constant, but similar facet-scale rms surface facet slopes, to surrounding terrain. Waveform analysis of altimetry returns reveals that nadir backscatter returns from basin floors are greater than nadir backscatter returns from basin surroundings and have narrower pulse widths. This suggests that floor deposits are structurally distinct from their surroundings, consistent with the interpretation that some of these basins may be filled with evaporitic and/or sedimentary deposits. Basin floor deposits also express a larger diffuse component to their backscatter, which is likely due to variations in subsurface structure or an increase in roughness at the wavelength scale [5]. We generate a high-resolution altimetry radargram of the T30 altimetry pass over an empty lake basin, with which we place geometric constraints on the basin's slopes, rim heights, and depth. Finally, the importance of these backscatter observations and geometric measurements for basin formation mechanisms is briefly discussed.
\end{abstract}

Citation: Hayes, A. G., et al. (2008), Hydrocarbon lakes on Titan: Distribution and interaction with a porous regolith, Geophys. Res. Lett., 35.

1. Background: On Titan, methane acts like water on Earth; raining out of the atmosphere, carving channels, and forming lakes in polar basins $[18,11]$. This methanebased hydrologic system drives many dynamic surface and

\footnotetext{
*Principal author

Email addresses: rjm342@cornell.edu (R.J. Michaelides), hayes@astro. cornell.edu (A.G. Hayes), marco.mastrogiuseppe@uniroma1.it (M. Mastrogiuseppe), zebker@stanford.edu (H.A. Zebker), thomas.g.farr@jpl.nasa.gov (T.G. Farr), michael.j.malaska@jpl.nasa.gov (M.J. Malaska), valerio.poggiali@uniroma1.it (V. Poggiali), jpm398@cornell.edu (J.P. Mullen)
}

atmospheric processes that generate geomorphologic structures: alluvial fans, river networks, and lakes, that are very similar to their terrestrial counterparts. Unlike Earth, Titan's surface liquids are restricted to the poles, where they collects in mare and lake basins. These basins chronicle the interaction between Titan's surface and atmosphere, and a more complete understanding of Titan's global hydrologic cycle necessitates an understanding of their physical properties.

Observed lacustrine features on Titan are found in varying states of liquid fill, that, at least in the south polar region, have been observed to change $[7,21]$. Lacustrine features are separated into 3 classes in accordance with Hayes et al. 2008: "dark" or filled lakes, "granular" or partiallyfilled lakes and/or mudflats, and "bright" or empty lake basins $[5,7]$. The off-nadir backscatter returns of "dark" lakes are consistently below the radar noise floor, and thus these features are interpreted as lakes or mare of standing liquid methane and ethane. "Granular" lakes exhibit brightness above the radar noise floor, but are still dark relative to their surroundings, and are interpreted as either shallow lakes that are completely penetrable by radar, or saturated regolith [5]. "Bright" lakes have the highest backscatter returns of all lacustrine features and are interpreted as 200-300 m depressions consistent with evaporated lakes [5]. Their observed morphology is accordant with evaporitic lake playas or karstic dissolution features such as dolines or poljes [4, 14]. Barnes et al. (2011) have shown that some of these features are correlated with high returns in the 5-micron window of the VIMS instrument, consistent with solid hydrocarbon evaporites. Additionally, their geographic association with filled lakes suggests that empty lake basins may be a transitional morphology of filled lakes that gradually lose their liquids via evaporation, surface runoff and/or subsurface infiltration [5]. This interpretation is consistent with the observation of temporal variation of south polar lake morphology due to changing liquid levels, and supports the theory of subsurface lake hydrologic networks linked to an underground alkanofer [5, 7, 21]. 
2. Methods: As of Titan Flyby T92 (7/10/2013), SAR and HiSAR coverage encompasses over $59 \%$ of Titan's surface, including $81 \%$ coverage in the North Pole and $67 \%$ coverage in the South Pole. This coverage, combined with accurate backscatter modeling of the SAR data, has allowed comprehensive mapping and analysis of different geomorphologic units on Titan's surface [9]. Titan's north pole has been observed repeatedly up to seven times in some locations, allowing for comparison of individual surface features from multiple viewing geometries (see figure 1). We take advantage of this repeat coverage to construct backscatter models of individual empty lake basins, and complement these models with the scattering behavior as observed by Cassini's RADAR instrument in altimetry mode. The difference in physical properties of these empty lake basins and their surroundings, as inferred from these complementary techniques, forms the focus of this work.

The Cassini RADAR instrument operates at $\mathrm{Ku}$ band $(\lambda=2.17 \mathrm{~cm})$ in four different modes: high-resolution imaging radar (SAR), lower resolution scatterometer, a microwave radiometer, and a profiling altimeter [2]. Any backscatter variations in SAR images are interpreted as changes in surface roughness on the scale of the $\mathrm{Ku}$ band wavelength, changes in surface dielectric properties, variations in subsurface structure, changes in incidence angle, or changes in the orientation of surface scattering facets at the pixel scale ( $300-2000 \mathrm{~m}$; [9]). Surfaces on Titan are brighter at larger incidence angles than Earth surfaces, but not any brighter at smaller incidence angles, thus necessitating a diffuse term of radar backscatter in addition to the specular return from Titan's surface. Due to the lowloss tangent of surface materials on Titan, diffuse scatter is partially attributable to subsurface structural heterogeneities [25]. In this work, we assume that the observed changes in backscatter magnitude from pass to pass are due primarily to incidence angle effects and not temporal change. Multipass analysis from similar terrain types on Titan vary systematically with angle; i.e. they always decrease with increasing angle, independent of the order in which the data are acquired. So temporal change on the surface of empty lake basins, physical or chemical, is likely not significant enough to account for backscatter variations observed from pass to pass. Also, since most observations are consistent with assuming some mixture of frozen (or liquid in lakes) hydrocarbons, and most solid hydrocarbons have dielectric constants not too far from 2, the large variations in backscatter intensity are not explained by small deviations in dielectric properties. We conclude that the observed variability is due primarily to incidence angle, roughness, and dielectric constant changes, in that order.

The SAR imaging mode has the highest resolution of all operational modes at $\sim 300 \mathrm{~m} /$ pixel, but each individual flyby is restricted to a narrow range of incidence angles $[25,7]$. The Cassini RADAR instrument's HiSAR mode images Titan's surface at a higher altitude, and consequently lower resolution $(\sim 1-5 \mathrm{~km})$ than the SAR mode.
However, HiSAR coverage is more extensive than SAR coverage, and can image the surface over a greater range of incidence angles during an individual observation.

Nadir-operating radar altimetry data provide constraints on surface topography, roughness, and effective dielectric constant $[12,13]$. By combining multiple SAR, HiSAR, and closest-approach altimetry observations, a wide range of viewing geometries can be investigated (angles of $0^{\circ}$ $64^{\circ}$ ) for specific lacustrine features at spatial scales of several $\mathrm{km}$, which is sufficiently fine to study the scattering properties of regions contained completely within the geomorphologic boundaries of individual empty lake basins.

Accurate backscatter profiles of both individual features and averaged geomorphologic units can be created by combining multiple SAR observations at different incidence angles. From radar altimetry, the nadir scattering properties of empty lake basins can be determined and compared to the inferred scattering properties from offnadir observations. By comparing the nadir and off-nadir scattering properties of empty lake basins and their immediate surroundings, the physical property differences between the two can be investigated.

Backscatter modeling was conducted over regions of Titan's north pole that have been imaged by SAR a minimum of three times (for proper parameter estimation from curve-fitting). In regions that have been imaged more than the minimum of three times, error analysis of backscatter models is also conducted. The backscatter modeling dataset used comprises T16-T92, and consists of 256 pixel-per-degree SAR images (oversampled above the intrinsic Cassini SAR resolution) of returned amplitude uncorrected for incidence angle variations. Geomorphologic mapping was conducted in Esri's ArcGIS with georeferenced SAR images at a 1:100,000 spatial resolution, and backscatter characteristics of empty lakes were determined with the ArcGIS Zonal Statistics tool. All data was exported to MathWorks's Matlab, where model fitting was conducted.

The Cassini RADAR instrument's altimetry mode has a main beam width $(-3 \mathrm{~dB})$ of 0.35 degrees and a $4.25 \mathrm{MHz}$ bandwidth which corresponds to a $35 \mathrm{~m}$ range resolution [13]. We processed T30 and T91 altimetry-mode observations through application of a Blackman taper function, range compression, incoherent averaging of pulses within each burst for pulse waveform analysis, and application of a super-resolution technique to extrapolate the bandwidth and increase range resolution (as in [12]). Variations in returned power due to changes in spacecraft altitude over transects of interest in this work are negligible, and attenuator levels are constant over our transects of interest. Thus we treat uncalibrated power as a proxy for backscattering variation. 


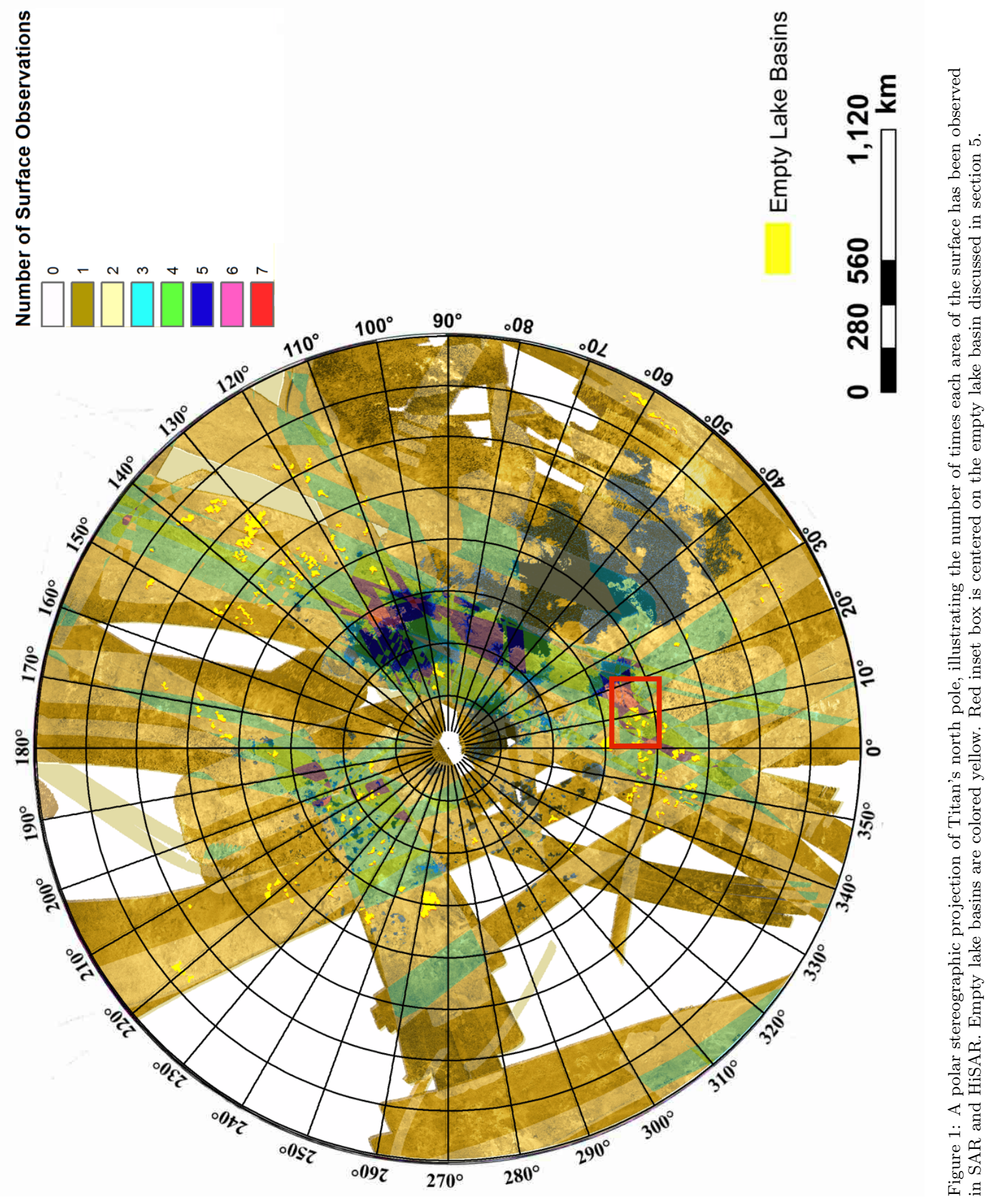


As with surface returns in the SAR operating mode, surface returns in altimetry mode are a function of surface roughness at the wavelength scale and surface bulk dielectric constant. Returned pulse power is a function of surface dielectric constant, and pulse width is related to surface roughness and volume scattering [13]. By comparing the shape of returned waveforms from different surfaces, we can investigate the relative differences in surface dielectric constant, surface roughness, and volume scattering. These results provide complementary information to SAR backscatter modeling and, when the two datasets are considered simultaneously, provide constraints on surface scattering properties, composition, and structure of investigated surfaces.

3. Derivation of SAR scattering model: Repeat SAR coverage of Titan's north polar region, complemented by altimetry observations, allows the ability to analyze the scattering properties of surface features over varying incidence angles, and temporal variations of surface features can be characterized, if present. By comparing observed backscatter profiles to scattering models, constraints on the effective physical properties of surface features can be placed, and the relative variation in the physical properties between surface features and their surrounding terrain can be quantified.

Our ability to infer physical and compositional properties of observed features, however, is dependent on the assumption that the assumed radar scattering model accurately represents Titan's surface. For solid surfaces, radar backscatter is modeled using a quasi-specular facet model, which models the surface as a series of planar facets oriented at varying angles with respect to the long-wavelength (footprint-scale) surface topography. Each facet is assumed to be smooth (at wavelength scale) and produce a specular reflection. These reflections sum to the observed normalized radar cross section $\left(\sigma_{0}\right.$, defined as the area of an isotropic scatterer required to yield the observed radar echo intensity, normalized by the area of the observed radar footprint) $[16]$.

Quasi-specular models relating $\sigma_{0}$ to the angle of incidence, $\theta$, have been used previously to infer the surface properties of terrestrial planets (Mercury, Venus, Earth, Mars, and the Moon) (see, for example, [16, 22]). Radar observations from most, if not all, parts of Titans surface require a diffuse scattering component in addition to the facet-scattering models used here [25, 24]. Zebker et al. (2008) further showed that a diffuse component is needed to obtain agreement between active radar and passive backscatter observations. This diffuse component can either be a multiple scattering interaction on the surface, or, more likely perhaps, a volume component enabled by Titans low-loss surface material. For this paper we concentrate on the specular component of the signal as this dominates over most of the incidence angles analyzed. Here we have examined only specular scattering mechanisms, and in particular those commonly denoted Gaussian, Hagfors, and exponential laws. This is in keeping with much of the planetary literature, as these models are simple and provide a valid comparison with other planetary surfaces that have been analyzed from data acquired by previous missions. The parameters of roughness and dielectric constant are not necessarily to be taken as literal, but rather as relative, as various locations are compared. Nearly all of the observed radar returns from Titan fit one of these laws functionally, with a diffuse component sometimes required [25]. In accordance with Wye et al. (2007), we use a $\cos ^{n}$ diffuse component. The diffuse backscatter observed on Titan is only weakly sensitive to the $n$ exponent in the diffuse backscatter mode. For the purposes of this work, we use an exponent value of $n=1.24$, which is within the range of exponent values reported in Wye et al. (2007), and do not vary $\mathrm{n}$ when fitting to observed backscatter profiles. As we are primarily interested in the relative differences in scattering behavior between empty lake basins and their immediate surroundings, and are not attempting to derive definitive surface properties, the weak sensitivity to $\mathrm{n}$ justifies this assumption. We also note here the necessity for a volume scattering component that can simultaneously fit Cassini passive radiometry data as well as active RADAR backscatter, although we do not attempt to derive such a scattering term in this work.

We fit observed backscatter profiles to models that are dependent upon the effective surface dielectric constant, surface rms facet slope angle (i.e., facet-scale roughness), and amplitude of the diffuse term. We use a LevenbergMarquardt least-squares minimization to find the best-fit model coefficients for the bright empty lake basins and their immediate surrounding terrain. The LevenbergMarquardt minimization is a standard curve-fitting technique that has been used previously to fit Cassini SAR backscatter values [6, 7]. After model inversion, dielectric constant, surface rms angle, and volumetric scattering coefficient can be extracted from the model best-fit coefficients.

In accordance with the Hagfors Model, we assume a gently undulating surface (Kirchoff approximation), and assert that the surface can be suitably defined by a surface facet orientation probability density function, and a surface height deviation probability function. For most surface backscatter models, a gaussian or exponential distribution is assumed for the facet orientation probability and height deviation probability functions [25]. Outlined below is Hagfors's equation relating $\sigma_{0}$ (which we will hereafter refer to as $\sigma_{q}$, the $\sigma_{0}$ value due to quasi-specular reflection) to the surface facet orientation and height deviation probability function.

Hagfor's specific radar cross section and probability 
density function:

$$
\begin{array}{r}
\sigma_{q H}=\frac{\varrho C}{2}\left(\cos ^{4} \theta+C \sin ^{2} \theta\right)^{-\frac{3}{2}} \\
C=\frac{\lambda^{2} R_{0}^{2}}{16 \pi^{2} h^{4}}=\frac{1}{\tan ^{2} \phi_{r m s}}
\end{array}
$$

Where $\sigma_{q}$ is the radar cross section, $\rho$ is the Fresnel power reflection coefficient for normal incidence, $\vartheta$ is the incidence angle, $\mathrm{C}$ is Hagfors' parameter for surface roughness, $R_{0}$ is the correlation length, $\mathrm{h}$ is the rms vertical surface height deviation and $\phi_{r m s}$ is the rms surface facet angle.

The Hagfors parameter has traditionally been inverted as in (9) to derive the rms surface facet slope angle. The Hagfors parameter is related to both the correlation length $R_{0}$ and the rms vertical surface height deviation $h$, and so placing a constraint on $\mathrm{h}$ requires a priori knowledge of the correlation length. Because of the fractal nature of surface roughness, there has been extensive work towards determining the proper correlation length for different terrestrial scattering surfaces, but there is no clear consensus on the proper correlation length for Titan's surface [23, 19]. Since we are not directly interested in absolute measurements of $\mathrm{h}$ in this work, we determined the upper and lower bounds for $\mathrm{h}$ by considering the end member cases of $R_{0}=\frac{6 \lambda}{2 \pi}$ (from the Kirchoff Approximation, where the wavelength $\lambda=2.17 \mathrm{~cm}$ ), and $R_{0}=6,000 \mathrm{~km}$ (the semimajor axis of the radar footprint).

Gaussian specific radar cross section and probability density function:

$$
\sigma_{q G}=\frac{\varrho C}{\cos ^{4} \theta} e^{-} \operatorname{Ctan}^{2} \theta
$$

Where $\sigma_{q}$ is the coherent radar cross section, $\vartheta$ is the incidence angle and $\phi_{r m s}$ is the rms surface facet slope angle.

Similarly, we can express the Exponential specific radar cross section and density functions:

$$
\sigma_{q E}=\frac{3 \rho C}{\cos ^{4} \theta} e^{-\sqrt{6 C} \tan \theta}
$$

These quasi-specular models can approximate the coherent (i.e. specular, small incidence angle) backscatter returns from planetary surfaces. However, an additional, diffuse term must be added to these scattering laws to account for the incoherent (i.e. diffuse, large incident angle) returns from the surface. A diffuse term modeled as $A \cos ^{n}$ (where A represents a volumetric scattering coefficient) has been widely used in Titan radar research, and is thus the diffuse term used in this work.

$$
\sigma_{d}=\operatorname{Acos}^{n} \theta
$$

So that the total backscatter radar cross-section is the sum of the quasi-specular cross-section and the diffuse cross-section.

$$
\sigma_{0}=\sigma_{q}+\sigma_{d}
$$

After applying a Levenberg-Marquardt least-squares bestfit to the observable data, model best-fit coefficients for $\rho$, $\theta$, and $\mathrm{A}$ are generated, from which dielectric constant, rms surface facet slope angle, and volumetric scatter coefficient can be extracted as follows:

$$
\begin{array}{r}
\epsilon=\left(\frac{1+\sqrt{\rho}}{1-\sqrt{\rho}}\right)^{2} \\
\tan \left(\phi_{r m s}\right)=\frac{1}{\sqrt{C}}
\end{array}
$$

The scattering models proposed here are simple and consistent with planetary radar tradition, yet they are derived from physical models that consider both physical structure and chemical makeup of the scattering surface. While these models are surely more simple than the actual physics of scattering from Titan's surface, without better constraints it is incredibly difficult to distinguish among many other approaches that vastly increase the number of parameters describing any given surface. Nonetheless it may be possible to extend these results by incorporating more variability in the amount of wave penetration through the low-loss Titan surface material, or by considering more recent surface scattering models (such as the IEM and POM as in [15]), and this is left for future work.

4. Observations and Interpretations: Observed $\sigma_{0}$ and incidence angle values from multiple SAR passes of an individual feature are fitted to the previously-mentioned quasi-specular scattering models using a Levenberg- Marquardt least-squares minimization to determine the bestfit values for the models' coefficients: dielectric constant, rms surface angle, and diffuse scattering coefficient. Results from modeling the backscatter curves of Titan's empty lake basins suggest that empty lake basins have a higher dielectric constant than their surroundings, but a similar rms facet slope angle to within error (68\% confidence interval; see figures 2 and 3). This is true regardless of which quasi-specular model (Hagfors, Gaussian, or Exponential) is used to fit the observations.

For any fixed incidence angle, backscatter is dominated by surface roughness and dielectric constant, and our model results predict no appreciable difference in rms facet slope angle between empty lake basins and their surroundings. Additionally, the reflectivity function of returned surface permittivity values from Titan's surface is highly nonlinear, which allows for a narrow range of surface dielectric constant values to potentially produce a large range of backscatter values. we suggest that the difference in offnadir backscatter returns between empty lake basins and 
their surroundings is due mainly to a difference in dielectric constant (and thus bulk composition) between empty lake basins and their surroundings, and/or changes in smallscale surface roughness (rather than facet-scale slope orientations, to which quasi-specular models are most sensitive; [3]). Furthermore, all three models predict a higher dielectric constant and diffuse amplitude for the basins than the surroundings, suggesting compositional variations between these surfaces, and either an increased volume scattering component or increased small-scale roughness (wavelengthscale as opposed to facet-scale).

We find that at nadir viewing geometries, received power of echo returns are higher from empty lake basins than from their surroundings, although the relative difference in power is more pronounced in SAR backscatter than altimetry backscatter (see figure 5). In nadir viewing geometries, returned power is a factor primarily of surface dielectric constant and surface roughness, although at nadir viewing geometries surface roughness has a less pronounced effect on returned power than in off-nadir viewing geometries [3]. Pulse-width analysis reveals that returns from basin floors are narrower than returns from basin surroundings, which suggests that basin floors are smoother than their surroundings at the meter scale, and/or that the surrounding terrain exhibits a larger penetration depth-and therefore a larger volume of subsurface heterogeneities-by the radar instrument (see figures 5 and 8 ).

Diffuse scattering encapsulates many different scattering mechanisms (wavelength-scale surface roughness, multiple scattering, and volume scattering), and similarly, altimeter pulse waveforms depend on several surface geophysical paramaters (dielectric constant, surface roughness, the vertical distribution of scatterers within an altimeter footprint, the penetration depth of the surface, etc). For a planetary surface whose physical properties are as poorly understood as Titan's, it is difficult to ascertain the absolute importance that any one of these physical surface paramaters may have on the bulk scattering behavior from any given region on Titan's surface. Furthermore, the significant differences in viewing geometry between the RADAR's altimeter mode and SAR mode introduce complications in comparing data acquired between these two operational modes. Nonetheless, we attempt to reconcile SAR and altimetry observations through interpretation of these datasets, which may explain the physical surface properties of Titan's empty lake basins and their surrounding terrain.

In SAR mode, empty lake basins have a higher $\sigma_{0}$ value than their surrounding terrain, and backscatter modeling suggests that this discrepancy is due primarily to differences in effective dielectric constant (and thus bulk material composition and/or porosity) rather than differences in surface roughness. In altimetry mode, larger backscatter returns and narrower pulse widths over empty lake basins further suggest that backscatter variations between empty lake basins and their surroundings are primarily attributable to variations in dielectric constant, al- though variations in wavelength-scale and meter-scale surface roughness are also likely present.

Altimeter pulse widths are narrower for returns from empty lake basins than from surrounding terrain, which suggests that empty lake basin floors may be smoother than their surrounding terrain at the meter-scale. However, because of the low-loss tangent of Titan surface materials, variations in altimeter pulse width may also be partially attributable to variations in penetration depth of the altimeter pulse (analagous to radar altimeter penetration of terrestrial ice and snow, as in [8]). Unfortunately, inadequate knowledge of the physical and chemical properties of Titan's surface materials precludes a complete understanding of the relative importances of surface roughness and penetration depth on the returned altimeter pulse width for a given surface. The interpretation that penetration depth (and therefore volume scattering) is significantly more important than surface roughness in determining returned altimeter pulse width would seemingly be at odds with the SAR backscatter modeling results presented above, as altimeter pulse widths are wider over surrounding terrain than over empty lake basin floors, while backscatter modeling suggests diffuse scattering is more pronounced over basin floors than over surrounding terrain. Additionally, the three-dimensional positions and orientations of surface and subsurface scatterers, the dependence of penetration depth on incidence angle, and the net effect of these properties on diffuse scattering, are all unknown. We hypothesize that there is a change in the surface roughness correlation length $\left(R_{0}\right.$ from equation $3)$, the standard deviation of vertical surface heights $\left(\sigma_{h}\right)$, and the penetration depth, between empty lake basins and their surroundings. That is, meter-scale surface roughness and penetration depth are both larger over surrounding terrain than over empty lake basin floors, but we do not fully understand the importance of these two properties on diffuse scattering from Titan's surface, and note that this is a topic that necessitates further inquiry.

Of note are several empty lake basins that appear distinctly different in observations acquired at different incidence angles. These "peekaboo lakes" seemingly disappear at large incidence angles, exhibiting scattering properties similar to their surroundings and different from conventional empty lake basins (see figure 4). Several of these features were suitably observed to allow the generation of backscatter models, and these models-unlike those of other conventional empty lake basins-exhibit a tangent point or secant point between the scattering curves of the features and their surroundings (see figure $3 \mathrm{~b}$ for a scattering model that almost crosses at $25^{\circ}$ ). That is, at small incidence angles, these features are brighter than their surroundings, but at larger incidence angles these features appear completely indistinct, within error, from their surroundings. There is a larger disparity between predicted rms facet slope angle of these features and their surroundings as compared to conventional empty lake basins, and this may partly explain the unique scattering behavior of these 


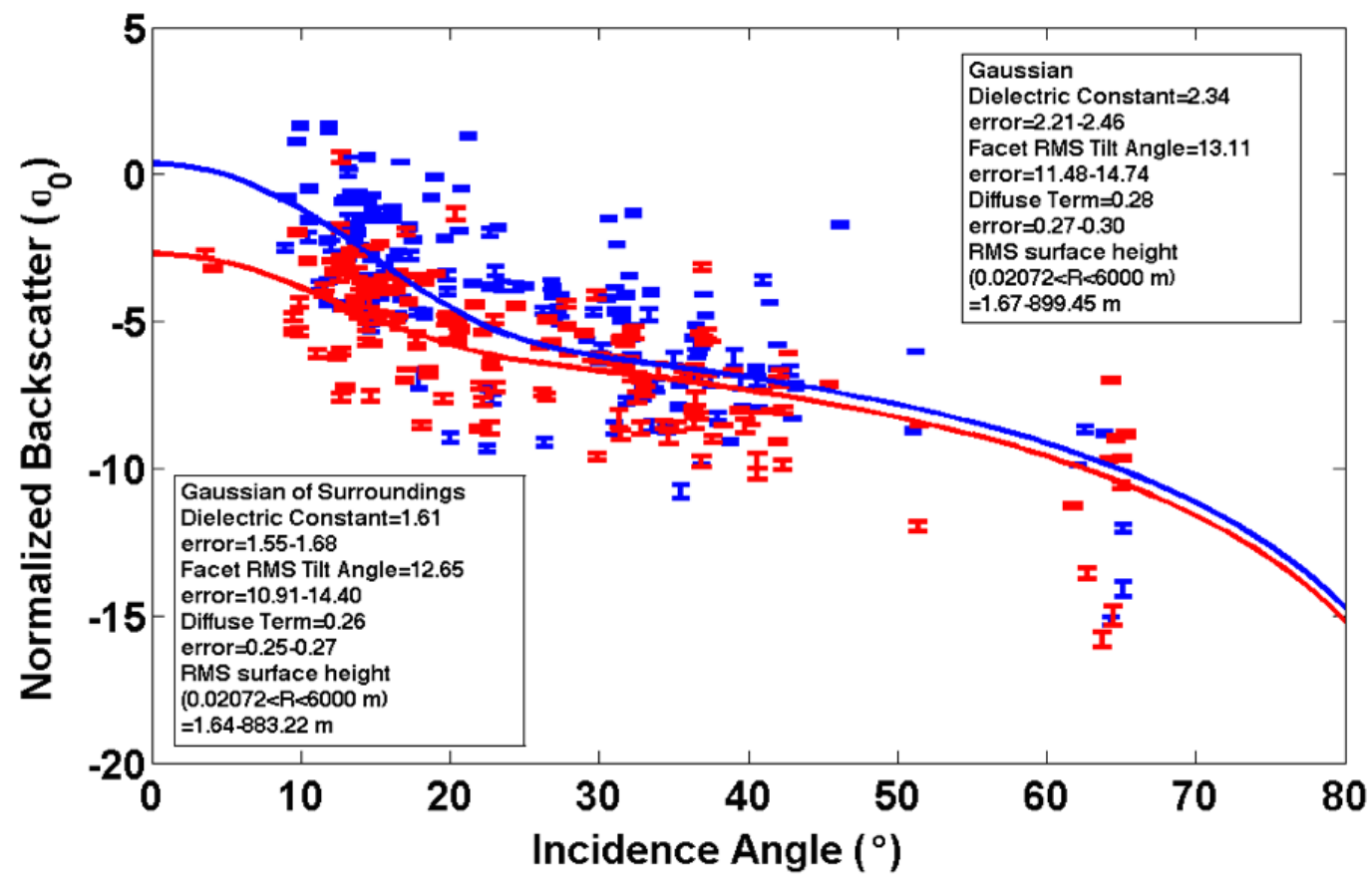

Figure 2: Backscatter curve of all north polar empty lake basins (blue data points, error bars, and line of best-fit) and their surrounding terrain (red data points, error bars, and line of best-fit). When modeled in bulk, Titan's north polar empty lake basins display the same off-axis scattering behavior as individual features. Inferred model best-fit coefficients also match the same trend as in individual features; empty lake basins have higher dielectric constant and volumetric scattering terms than their surroundings, but similar rms surface facet slopes.
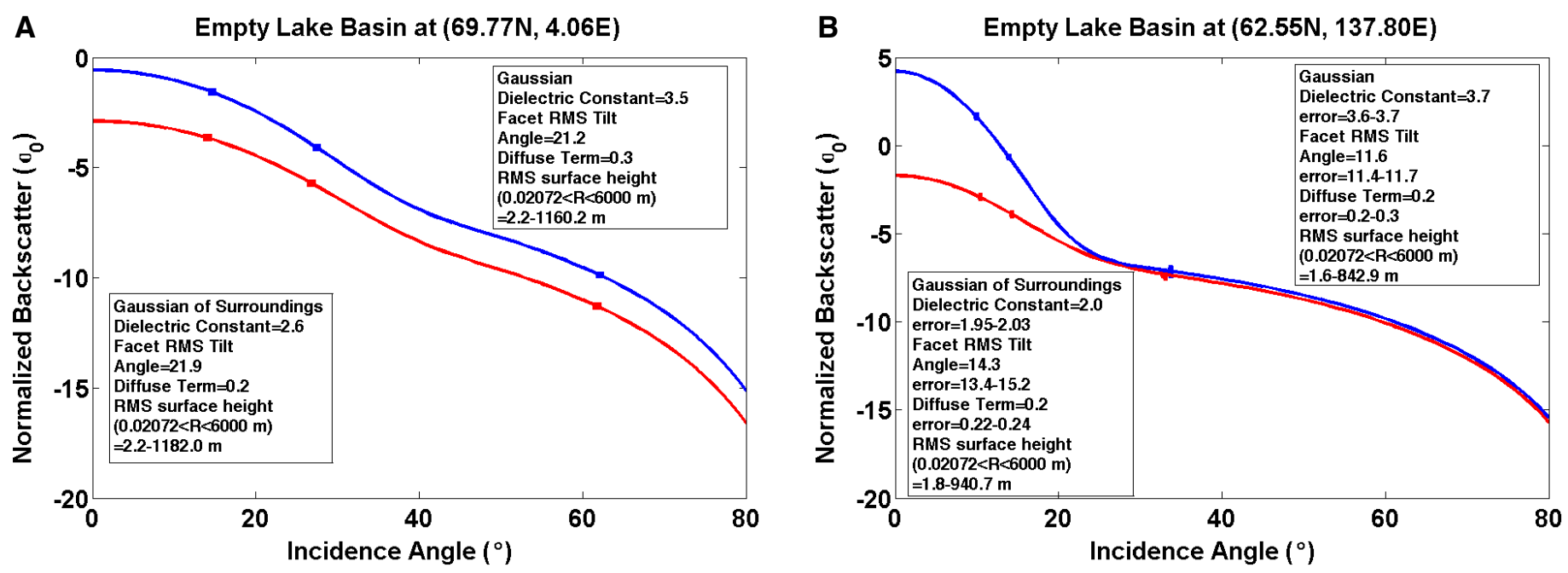

Figure 3: Backscatter curves of empty lake basins (blue), and their surroundings (red); These curves illustrates the general trend for all empty lake basins: a higher dieletric constant and volumetric scattering term, but similar rms surface facet slopes, relative to their surroundings. Note the tangent point between the red and blue model curves in figure 3B; at larger incidence angles, the empty lake basin is largely indistinguishable from its surroundings. 


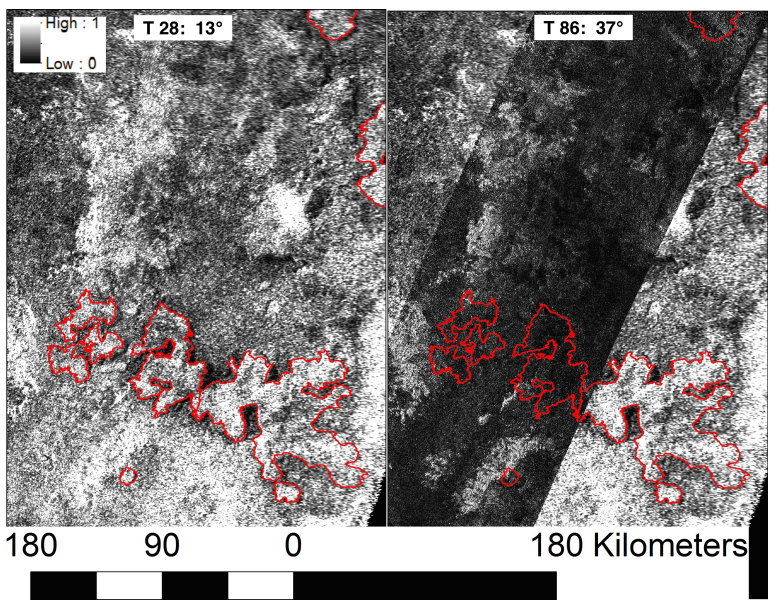

Figure 4: A cluster of north polar "peekaboo" lakes. Note the change in backscatter behavior from low incidence angle (left) to high incidence angle (right). Both images are of $\sigma_{0}$ uncorrected for incidence angle effects.

features. The unique scattering behavior of these features may also be due, in part, to surface liquid saturation levels; if the near-surfaces of these features have higher liquid saturation levels than other empty lake basins, the relative difference in dielectric constant between near-surface host material and void space would be less pronounced than in other empty lake basins, potentially explaining the lower brightness of these features at certain off-nadir viewing geometries. Unfortunately, no altimetry passes over these features currently exist. Future studies into the scattering behavior and physical properties of these features will be worthwhile if more observations of them become available. At present we will merely note that our results suggest that these features may be distinct from conventional empty lake basins, and may represent a unique type of empty lake basin.

5. Lake Morphology: After applying the superresolution techniques described in [12] to the T30 altimetry pass, we generated a radargram over an empty lake basin transected by the aforementioned altimetry pass. We employed a doppler processing technique that applies offnadir, antenna gain pattern, and time-delay corrections to the altimetry datasets, and incoherently averages the overlapping doppler filters of successive altimetry bursts (as in [17]). This processing technique improves the along-track altimetry resolution to a final resolution of $600 \mathrm{~m}$ (see figure $6 \mathrm{~A}$ for the final radargram, and $6 \mathrm{~B}$ for a topographic profile generated by a pixel-tracking algorithm).

Through complimentary analysis of a high resolution radargram of the T30 altimetry transect with super-resolved waveform analysis, we are able to construct a first-order topographic transect of the empty lake basin located at $69.77 \mathrm{~N}, 4.06 \mathrm{E}$ (see figure 6). By measuring the pulse timedelay among pulses in multi-peaked altimetry waveforms, we are able to measure the offset in elevation between surface reflectors (the basin's raised rim, surrounding terrain, and basin floor, in the case of the altimetry footprints on the edge of an empty lake; see figure 8). We find that the empty lake basin located at $(69.77 \mathrm{~N}, 4.06 \mathrm{E})$ has raised rims of $\sim 200-300 \mathrm{~m}$, and an absolute depth that varies from $\sim 100-350 \mathrm{~m}$.

First-order basin slopes can be calculated for regions of the empty lake basin where the change in elevation occurs in the along-track direction; at present we do not discuss basin slopes in the azimuth direction (such as the altimetry bursts centered on the inbound edge of the empty lake basin, see Figure 6 inset) due to associated delay/elevation ambiguities in the azimuth direction (see [17]). First-order basin slopes along the outbound basin edge can are calculated, as the variation in elevation along this edge is in the along-track direction. The exterior basin slope is $\geq 22.8^{\circ}$, while the $\geq 35.7^{\circ}$ interior slope is significantly larger. These slope measurements represent lower-bound measurements, as the total along-track components of these basin slopes may be smaller than the along-track resolution of the radargram. In fact, returns from the basin floor, raised rim, and surrounding terrain are all resolved within a single $600 \mathrm{~m}$ doppler cell after advanced processing of altimetry data (see radargram in figure 6), which suggests that this interior slope is $45.3^{\circ}$ or larger. We report a lower-bound estimate of $\geq 45.3^{\circ}$ for this interior slope, noting that the actual slope may be steeper than this value, as the actual along track distance from basin floor to raised rim may be less than $600 \mathrm{~m}$. The steep nature of the interior slopes of this empty lake basin may be indicative of a karst-style formation mechanism, such as dissolution and collapse of the surface or suffosion, whereby fine-grained surface materials are gradually permeated through a porous subsurface [4].

We also note that after applying superresolution techniques (as in [12]) to the T30 altimetry track, several pulse waveforms corresponding to altimetry returns over the surrounding terrain of the empty lake at $(69.77 \mathrm{~N}, 4.06 \mathrm{E})$ exhibit a unique waveform signature. The main peak of each of these altimetry waveforms is double-peaked; that is, after applying superresolution techniques, we are able to resolve that the main peak of these altimery returns is composed of two smaller peaks seperated in range by no more than $83 \mathrm{~m}$ (upper bound is dictated by the speed of light in a vacuum; see figure 7). This scattering behavior may be suggestive of a surface with two dominant surface elevations (possible surface geometries are illustrated in figure $7 \mathrm{~A}-7 \mathrm{C}$ ). Investigation of complementary SAR images in this region does not reveal any macroscale breaks in surface slope within altimetry footprints corresponding to double-peaked returns, which suggests that the situation outlined in figure 7A is likely not responsible for observed double-peaked waveforms. Complimentary SAR image-analysis cannot rule out the possibility of subresolution breaks in surface elevation (as in 7B), and we note that this geometry may be responsible for the observed double-peak altimetry waveforms. However, if this double-peak scattering behavior cannot be attributable to 

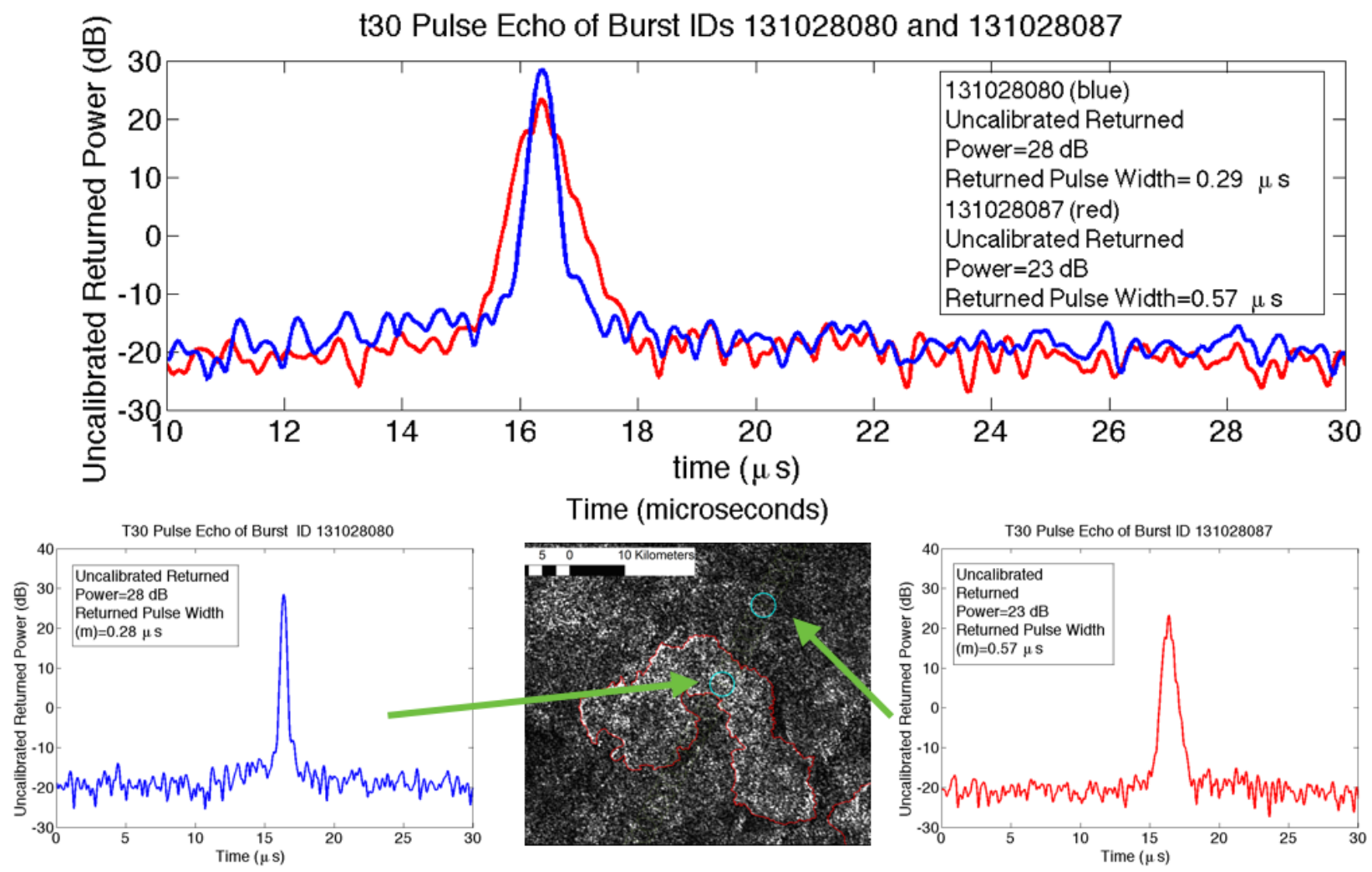

Figure 5: A waveform from a burst centered over the empty lake basin (blue), and a waveform from a burst centered over the empty lake basin's surrounding terrain (red; these two pulses are superimposed over each other for pulse width comparison). Note the higher return and narrower pulse width of the waveform of the burst centered over the basin floor; these features are indicative of a higher dielectric constant and smoother wavelength-scale roughness, respectively, for the empty lake basin floor. 

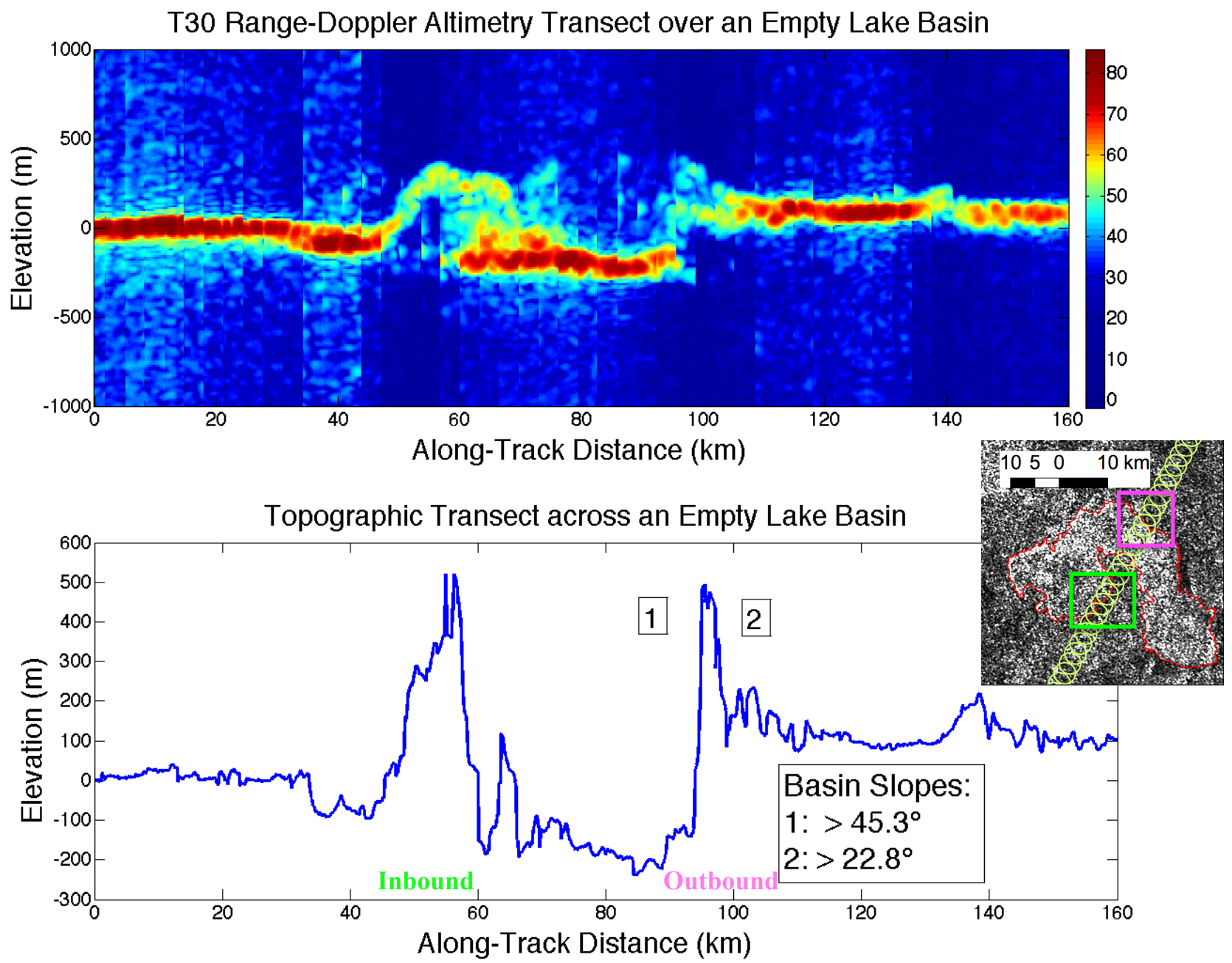

Figure 6: A: Improved radargram over empty lake basin at (69.77N, 4.06E) obtained by incoherent averaging of overlapping doppler bins (along-track spatial resolution of $600 \mathrm{~m}$, with a multi-look factor of 4 ). B: Topographic profile over empty lake basin obtained by a pixeltracking algorithm applied to the above radargram. Raised rim height, depression depth, and basin slopes were derived from processed individual altimetry waveforms (see figure 8), and from this radargram. The lower-bound measurements of basin slopes are numbered and labeled. Analysis of individual triple-peaked altimetry waveforms centered over slope 1 in conjunction with the processed altimetry radargram yields a slope of $\geq 45.3^{\circ}$ for this interior basin slope. Inset: SAR image of the empty lake basin, with altimetry bursts outlined in yellow. Inbound basin edge is surrounded by a green box, while the outbound basin edge is surrounded by a magenta box. 
sub-resolution surface scattering phenomena, it is possible that the Cassini instrument has sounded within the near surface, and resolved a subsurface return in addition to a surface return. The ability to use the Cassini radar to sound Titan mare has already been demonstrated [12], and other authors have noted the low-loss tangent of $\mathrm{Ti}-$ tan's surface materials [20], the possibility of subsurface structural horizons analogous to terrestrial caliches [10], and the likelihood of penetration of Titan's surface by the Cassini RADAR instrument [25]. If this double-peak scattering behavior is consistent with surface penetration by the Cassini RADAR instrument, this may represent a direct measurement of a subsurface sedimentary layer or karstic dissolution void-space in Titan's subsurface. We note that this result is dependent upon adequate demonstration that sub-resolution surface scattering geometries (as in 9B), along with other surface scattering geometries, cannot explain these double-peaked altimetry waveforms, but regardless, this observation demands future investigations into the potential subsurface sounding capability of the Cassini RADAR instrument.

6. Summary: The results obtained by quasi-specular backscatter modeling and altimetry waveform analysis suggest that, relative to their surroundings, empty lake basins have a higher surface dielectric constant, similar rms facet slope angle, smoother small scale roughness, and either a larger subsurface effective dielectric constant and/or porosity, or a smaller penetration depth. This is consistent with a previous analysis of the T30 altimetry pass of several empty lake basins, which ruled out differences in 2-cm wavelength-scale surface roughness as the sole cause for this disparity between the backscatter returns of empty lake basins and their surroundings [5]. Increased volumetric scattering is consistent with an increased number of preferentially oriented subsurface scatterers, increased void space and porosity, and/or larger differences in dielectric constant between the subsurface material and void space. The heterogeneous subsurface structure of empty lake basins may be consistent with karst-style dissolution and/or suffosion of Titan's regolith via interaction with liquid hydrocarbons [7, 14]. The correlation of some empty lake basins with 5 -micron bright evaporites suggests that post-formation, these features may be filled with evaporitic hydrocarbons from transient lake surface evaporation [1]. However, we note that not all empty lake basins are associated with this 5-micron bright spectral feature, and so the higher surface dielectric constant of empty lake basin floors may not necessarily be attributable to spectrally distinct evaporites. We have observed two unique classes of empty lake features based on scattering behavior, and note that additional subgroups of empty lake basins may be possible. We note that further analysis into the composition of Titan's empty lake basin floors is necessary to place better constraints on the composition of Titan's empty lake basins.

Through generation and analysis of a high resolution radargram transect over an empty lake basin, we have placed lower-bound estimates on basin depths, basin rim heights, and basin rim slopes, finding that interior basin slopes are as steep as $45^{\circ}$, and potentially steeper. The basin slopes generated from this altimetric profile suggest that karst-style dissolution of Titan's regolith is a potential formation mechanism for Titan's lake basins. We also report observations of double-peaked altimetry waveform returns over several regions of terrain surrounding an empty lake basin. This unique waveform may be indicative of sub-resolution breaks in surface elevation, or sounding of the near subsurface and reflection from a subsurface reflector, such as a subsurface stratigraphic horizon or caliche.

Future work will attempt to use the derived physical constraints placed on Titan's empty lake basins to explore possible formation mechanisms. A more detailed investigation into the likely compositions of empty lake basin floors, and whether or not variations in composition can explain the difference in scattering behavior between observed subclasses of empty lake basins, is needed. Preliminary estimates of basin rim slope can be incorporated with range-doppler analysis of altimetry waveforms to provide tighter constraints on the actual slope values of the T30 empty lake basin and other empty lake basins. This analysis will help to determine how empty lake basins form, and provide insight into their role in Titan's global methanebased hydrologic cycle.

Acknowledgements: The authors would like to thank Cornell University, the Jet Propulsion Laboratory, the Cassini Radar Data Team and the Cassini Engineering Team. We also thank Ralph Lorenz and one anonymous reviewer for their insightful comments during the review process. 

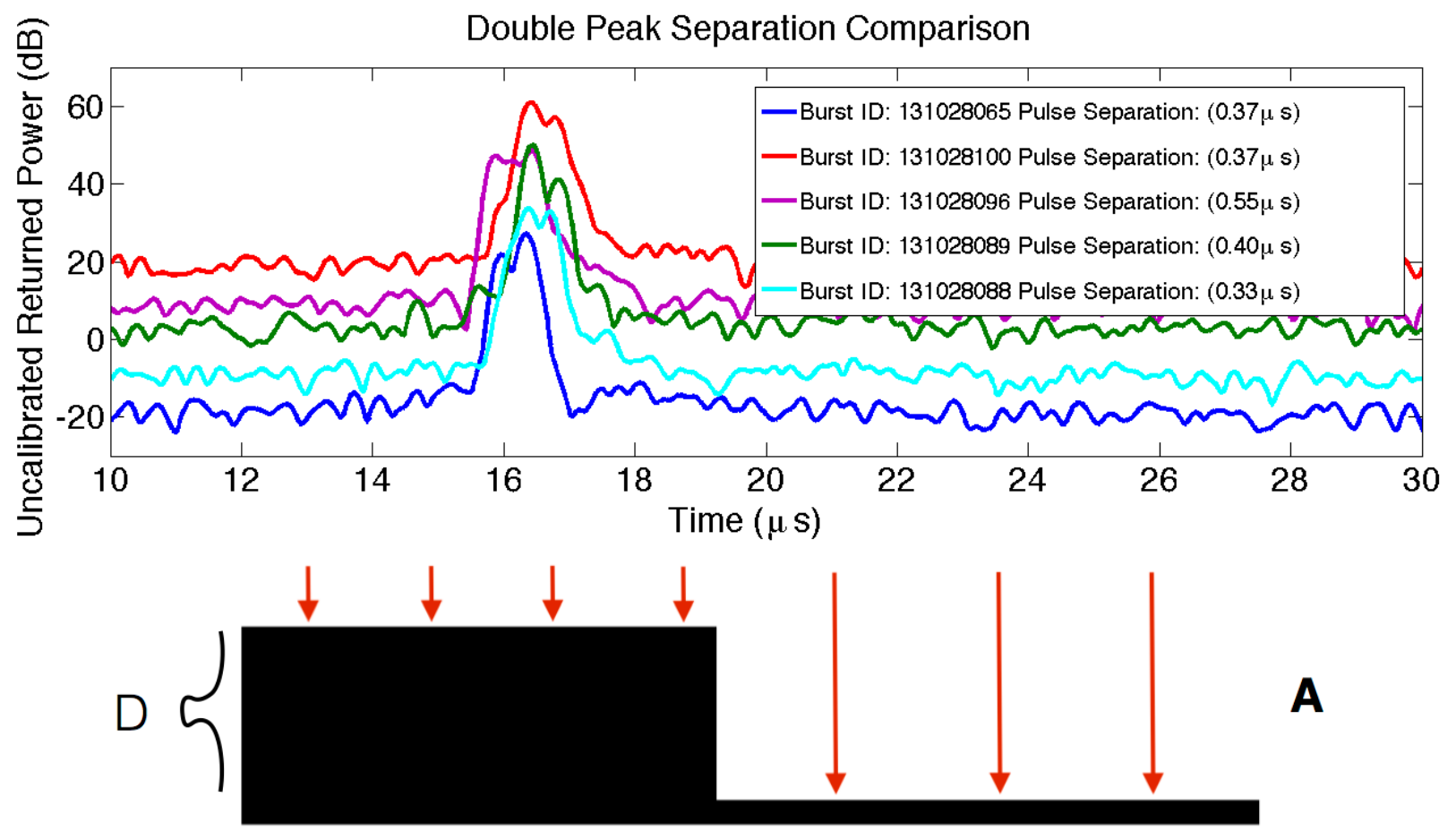

A
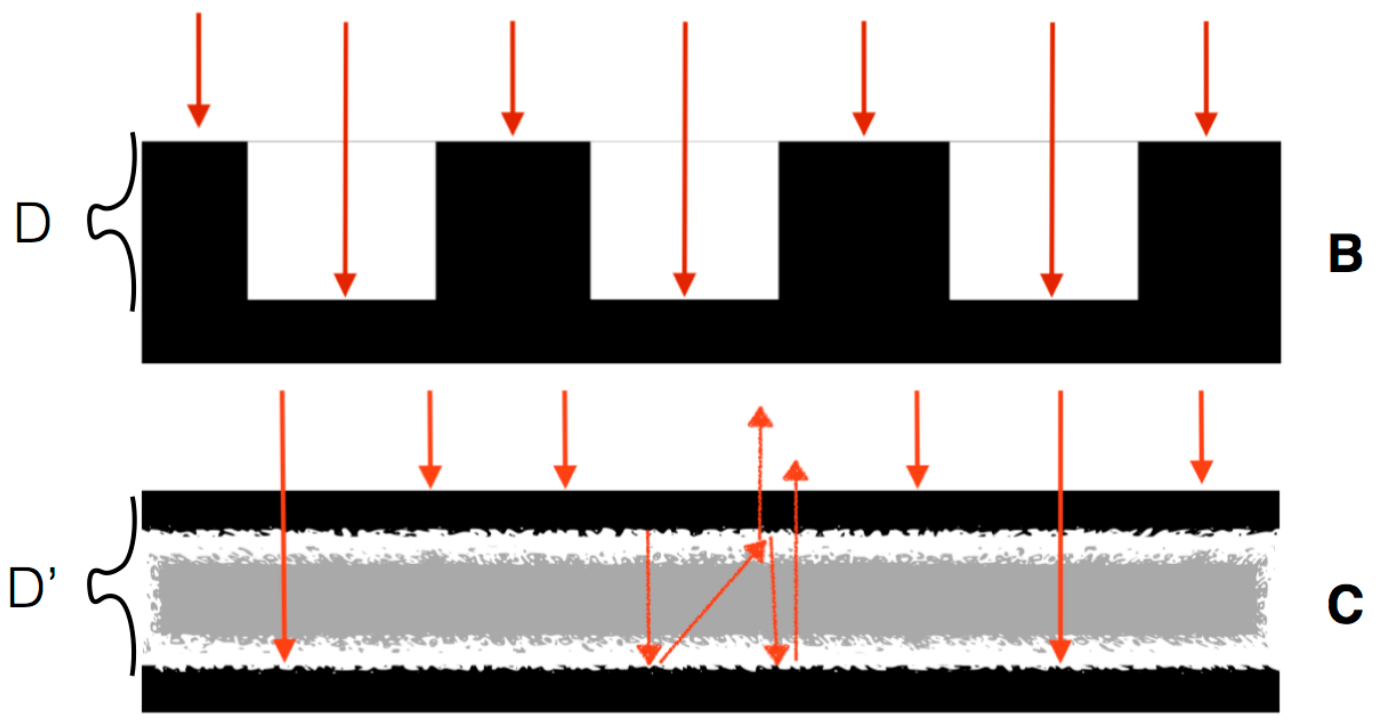

Figure 7: An example of several double-peaked altimetry pulse waveforms. Each successive waveform is offset by $10 \mathrm{~dB}$; corresponding Burst IDs and the distance between each waveform's double peaks are displayed in the legend. Below are three possible surface geometries that could produce a double-peaked waveform (downward-pointing arrows represent the incoming altimeter pulse; the horizontal dimension represents the resolution of a single altimetry footprint projected onto the surface of Titan). A: returns from two levels of constant elevation offset by distance D. B: "pock-marked" terrain of alternating sub-resolution plateaus and flats offset by distance D. C: A subsurface layer a distance $D^{\prime}\left(D^{\prime}=n^{*} D\right.$, where $\mathrm{n}$ is the index of refraction of the layer between each scattering layer) below the surface. The two peaks in each waveform would correspond to returns from the surface and the dominant subsurface layer. The index of refraction of this subsurface medium will affect wave propagation, and thus measured distances; therefore we assume an index of refraction of 1.0 for this work, noting that the true index of refraction of a subsurface medium would be larger, and therefore all measured distances reported in this work are upper bounds. Volume scattering between these two layers can introduce multiple reflections in this configuration. 

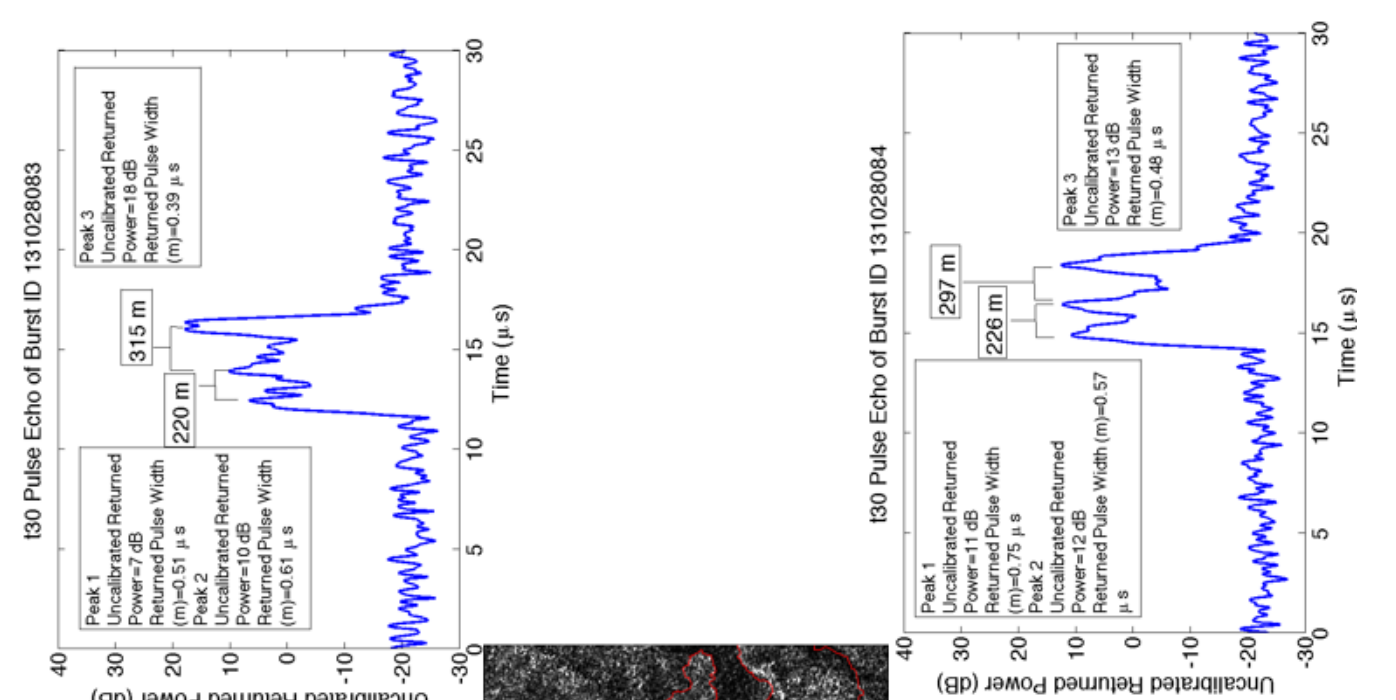

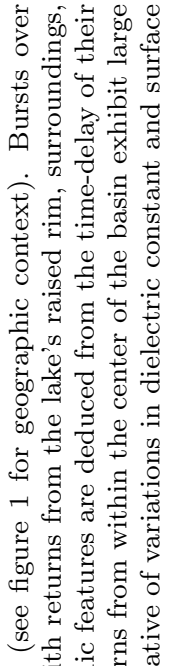

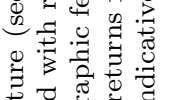

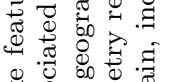

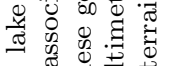

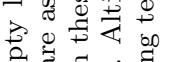

द्वे

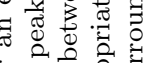

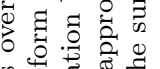

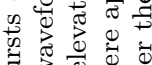

30 .

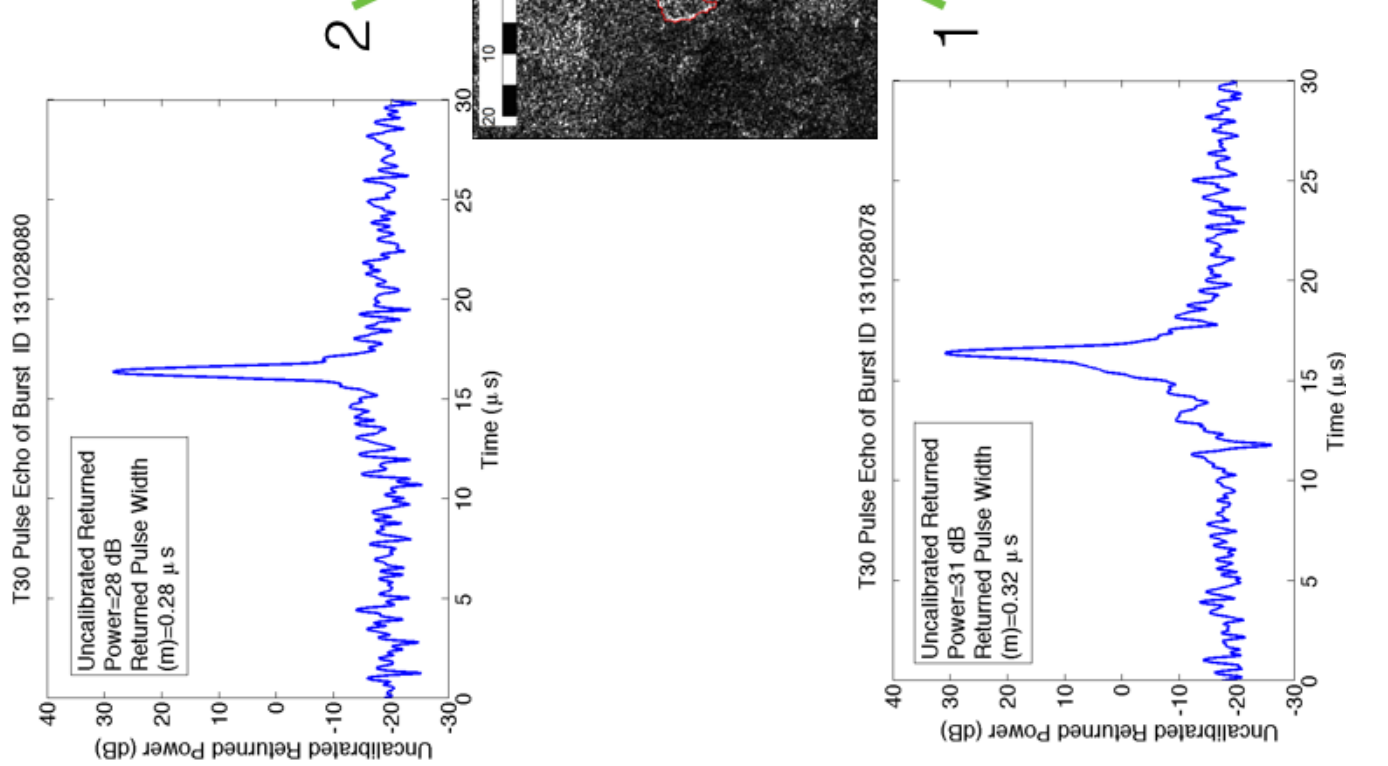

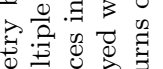

兽声过

ণิ

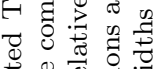

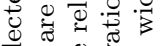

क.

है

o 0

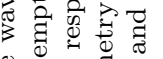

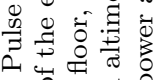

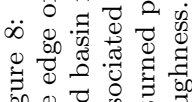

แn 


\section{References:}

[1] J. W. Barnes, J. Bow, J. Schwartz, R. H. Brown, J. M. Soderblom, A. G. Hayes, G. Vixie, S. Le Moulic, S. Rodriguez, C. Sotin, R. Jaumann, K. Stephan, L. A. Soderblom, R. N. Clark, B. J. Buratti, K. H. Baines, and P. D. Nicholson. Organic sedimentary deposits in titan's dry lakebeds: Probable evaporite. Icarus, 216.1:136-40, 2011.

[2] C. Elachi, M. D. Allison, L. Borgarelli, P. Encrenaz, E. Im, M. A. Janssen, W. T. K. Johnson, R. L. Kirk, R. D. Lorenz, J. I. Lunine, D. O. Muhleman, S. J. Ostro, G. Picardi, F. Posa, C. G. Rapley, L. E. Roth, R. Seu, L. A. Soderblom, S. Vetrella, S. D. Wall, C. A. Wood, and H. A. Zebker. Radar: The cassini titan radar mapper. Space Science Reviews, 115(1-4):71-110, 2004

[3] T. G. Farr. Radar Interactions With Geologic Surfaces. Guide to Magellan Image Interpretation. National Aeronautics and Space Administration, Jet Propulsion Laboratory, California Institute of Technology, Pasadena, CA, 1993.

[4] D. C. Ford, A. N. Palmer, and W. B. White. Landform Development; Karst. Geological Society of America Print, Boulder, 1988.

[5] A. G. Hayes, O. Aharonson, P. Callahan, C. Elachi, Y. Gim, R. Kirk, K. Lewis, R. Lopes, R. Lorenz, J. Lunine, K. Mitchell, G. Mitri, E. Stofan, and S. Wall. Hydrocarbon lakes on titan: Distribution and interaction with a porous regolith. Geophysical Research Letters, 35:9, 2008.

[6] A. G. Hayes, A. S. Wolf, O. Aharonson, H. Zebker, R. Lorenz, R. L. Kirk, P. Paillou, J. Lunine, L. Wye, P. Callahan, S. Wall, and Sep Elachi, C. Bathymetry and absorptivity of titan's ontario lacus. Journal of Geophysical Research (Planets), 115:9009, 2010.

[7] A.G. Hayes, O. Aharonson, J. I. Lunine, R. L. Kirk, H. A. Zebker, L. C. Wye, R. D. Lorenz, E. P. Turtle, P. Paillou, G. Mitri, S. D. Wall, E. R. Stofan, K. L. Mitchell, and C. Elachi. Transient surface liquid in titan's polar regions from cassini. Icarus, 211.1:655-71, 2011

[8] B. Legresy, F. Papa, F. Remy, G. Vinay, M. van den Bosch, and O. Z. Zanife. ENVISAT radar altimeter measurements over continental surfaces and ice caps using the ICE-2 retracking algorithm. Remote Sensing of Environment, 95:150-163, 2005.

[9] R. M. C. Lopes, E. R. Stofan, R. Peckyno, J. Radebaugh, K. L. Mitchell, G. Mitri, C. A. Wood, R. L. Kirk, S. D. Wall, J. I. Lunine, A. G. Hayes, R. Lorenz, T. Farr, L. Wye, J. Craig, R. J. Ollerenshaw, M. Janssen, A. Legall, F. Paganelli, R. West, B. Stiles, P. Callahan, Y. Anderson, P. Valora, and L. Soderblom. Distribution and interplay of geologic processes on titan from cassini radar data. Icarus, 205.2:540-58, 2010.

[10] R. D. Lorenz and J. I. Lunine. Erosion on titan: Past and present. Icarus, 122(1):79-91, 1996.

[11] J. I. Lunine and R. D. Lorenz. Rivers, lakes, dunes, and rain: Crustal processes in titan's methane cycle. Annual Review of Earth and Planetary Sciences, 37(1):299-320, 2009.

[12] M. Mastrogiuseppe, V. Poggiali, A. G. Hayes, R. D. Lorenz, J. I. Lunine, G. Picardi, R. Seu, E. Flamini, G. Mitri, C. Notarnicola, P. Paillou, and H. A. Zebker. The bathymetry of a titan sea. Geophysical Research Letters, 41.5:1432-437, 2014

[13] M. Mastrogiuseppe, V. Poggiali, R. Seu, R. Martufi, and C. Notarnicola. Titan dune heights retrieval by using cassini radar altimeter. Icarus, 230:191-97, 2014.

[14] K. L. Mitchell, M. J. Malaska, D. G. Horvath, and J. C. Andrews-Hanna. Karstic Processes on Earth and Titan. 45th Lunar and Planetary Science Conference (LPSC), the Woodlands TX. 2014.

[15] Ph. Paillou, D. Bernard, J. Radebaugh, R. Lorenz, A. Le Gall, and T. Farr. Modeling the $\{\mathrm{SAR}\}$ backscatter of linear dunes on earth and titan. Icarus, 230:208 - 214, 2014

[16] G. H. Pettengill. Physical properties of the planets and satellites from radar observations. Ann. Rev. Astron. Astrophys., 16:265, 1978.

[17] R.K Raney. The delay/doppler radar altimeter. IEEE Trans.
Geosci. Remote Sensing IEEE Transactions on Geoscience and Remote Sensing, 36.5:1578-588, 1998.

18] E. R. Stofan, C. Elachi, J. I. Lunine, R. D. Lorenz, B. Stiles, K. L. Mitchell, S. Ostro, L. Soderblom, C. Wood, H. Zebker, S. Wall, M. Janssen, R. Kirk, R. Lopes, F. Paganelli, J. Radebaugh, L. Wye, Y. Anderson, M. Allison, R. Boehmer, P. Callahan, P. Encrenaz, E. Flamini, G. Francescetti, Y. Gim, G. Hamilton, S. Hensley, W. T. K. Johnson, K. Kelleher, D. Muhleman, P. Paillou, G. Picardi, F. Posa, L. Roth, R. Seu, S. Shaffer, S. Vetrella, and R. West. The lakes of titan. Nature, 445.7123:61-64, 2007.

[19] A. K. Sultan-Salem. Fractal surfaces and the kirchhoff approximation for modeling quasi-specular surface scattering: Theory and applications. Ph.D. thesis, Stanford University, pages 7993, 2006.

[20] W. R Thompson and S. W. Squyres. Titan and other icy satellites: Dielectric properties of constituent materials and implications for radar sounding. Icarus, 86.2:336-54, 1990.

[21] E. P. Turtle, J. E. Perry, A. S. McEwen, A. D. DelGenio, J. Barbara, R. A. West, D. D. Dawson, and C. C. Porco. Cassini imaging of titan's high-latitude lakes, clouds, and south-polar surface changes. Geophysical Research Letters, 36(2), 2009. L02204.

[22] G. L. Tyler, R. A. Simpson, M. J. Maurer, and E. Holmann. Scattering properties of the venusian surface: Preliminary results from magellan. Journal of Geophysical Research: Planets, 97(E8):13115-13139, 1992.

[23] F. T. Ulaby. Microwave remote sensing. Reading, MA: AddisonWesley, 1982

[24] L. Wye, H. A. Zebker, R. M. Lopes, R. Peckyno, Le Gall, and M. A., Janssen. A backscatter analysis of titan's surface features and their global distribution using cassini radar data. American Astronomical Society, 40th DPS Meeting. Abstract, 31:22, 2008.

[25] L. C. Wye, H. A. Zebker, S. J. Ostro, R. D. West, Y. Gim, R. D. Lorenz, and The Cassini Radar Team. Electrical properties of titan's surface from cassini radar scatterometer measurements. Icarus, 188.2:367-85, 2007. 